\title{
Anomalous wave statistics following sudden depth transitions: application of an alternative Boussinesq-type formulation
}

\author{
Paul A. J. Bonar ${ }^{1}$ (D) Colm J. Fitzgerald ${ }^{2}$. Zhiliang Lin $^{3}$ - Ton S. van den Bremer ${ }^{4,5}$. Thomas A. A. Adcock ${ }^{4}$ \\ Alistair G. L. Borthwick ${ }^{1,6}$
}

Received: 17 August 2020 / Accepted: 12 March 2021 / Published online: 21 April 2021

(c) The Author(s) 2021

\begin{abstract}
Recent studies of water waves propagating over sloping seabeds have shown that sudden transitions from deeper to shallower depths can produce significant increases in the skewness and kurtosis of the free surface elevation and hence in the probability of rogue wave occurrence. Gramstad et al. (Phys. Fluids 25 (12): 122103, 2013) have shown that the key physics underlying these increases can be captured by a weakly dispersive and weakly nonlinear Boussinesq-type model. In the present paper, a numerical model based on an alternative Boussinesq-type formulation is used to repeat these earlier simulations. Although qualitative agreement is achieved, the present model is found to be unable to reproduce accurately the findings of the earlier study. Model parameter tests are then used to demonstrate that the present Boussinesq-type formulation is not well-suited to modelling the propagation of waves over sudden depth transitions. The present study nonetheless provides useful insight into the complexity encountered when modelling this type of problem and outlines a number of promising avenues for further research.
\end{abstract}

Keywords Rogue wave $\cdot$ Freak wave $\cdot$ Boussinesq-type equations $\cdot$ Skewness $\cdot$ Kurtosis

\section{Introduction}

Long considered the stuff of legend, rogue waves are now recognised as a serious hazard to ships and offshore structures. Historical reports of giant, powerful waves appearing first without warning and then suddenly vanishing have since been supported by theory and experiment (Dysthe et al. 2008;

Thomas A. A. Adcock

thomas.adcock@eng.ox.ac.uk

1 School of Engineering, The University of Edinburgh, Mayfield Road, Edinburgh EH9 3FB, UK

2 Inland Fisheries Ireland, 3044 Lake Drive, Citywest Business Campus, Dublin D24 Y265, Ireland

3 School of Naval Architecture, Ocean, and Civil Engineering, Shanghai Jiao Tong University, Shanghai 200240, China

4 Department of Engineering Science, University of Oxford, Parks Road, Oxford OX1 3PJ, UK

5 Faculty of Civil Engineering and Geosciences, Delft University of Technology, Delft 2628CD, The Netherlands

6 School of Engineering, Computing, and Mathematics, University of Plymouth, Drake Circus, Plymouth PL4 8AA, UK
Kharif et al. 2009). In recent decades, numerous studies have explored both the physical mechanisms which might produce such waves and the statistical parameters that may be used to estimate their occurrence probability. Comprehensive reviews are provided by Dysthe et al. (2008), Kharif et al. (2009), Slunyaev et al. (2011), Onorato et al. (2013), and Adcock and Taylor (2014), amongst others.

Rogue waves are typically defined as those having heights which are more than twice the local significant wave height (e.g. Holthuijsen 2007) but their study is complicated by a limited number of real-world measurements (Kharif et al. 2009) and conflicting views as to how much information can be inferred from these (Dysthe et al. 2008). The key question at present is whether such observations represent 'classical' extremes which can be described by conventional models and statistics, or 'freak' waves requiring new theories and approaches (Haver and Andersen 2000; Dysthe et al. 2008; Kharif et al. 2009). Some authors take the view that rogue waves are rare instances of random superposition in seas of weakly nonlinear waves (Christou and Ewans 2014; Fedele et al. 2016) whilst others hypothesise that certain waves, such as the well-known Draupner wave, must have been produced by some other 
forcing mechanism (Adcock et al. 2011; Cavaleri et al. 2016).

Other possible rogue wave generating mechanisms include modulational instability; interactions with variable bathymetry, opposing currents, or between crossing seas; wind forcing; or some combination of these factors (Dysthe et al. 2008; Kharif et al. 2009; Onorato et al. 2013; Fedele et al. 2016). Attempts to derive a single, unifying theory are complicated by the facts that geometric focusing cannot explain the transient nature of rogue waves (Janssen and Herbers 2009), that modulational instability requires an improbable set of initial conditions (deep-water waves with a narrow spectral bandwidth and narrow directional spreading) (Dysthe et al. 2008), and that rogue waves can be produced even when several of the foregoing factors are absent (Mori and Janssen 2006; Kharif et al. 2009).

The simplest theory assumes that the dynamics of ocean surface waves are purely linear, that the free surface elevation is a stationary, Gaussian process, and that the wave amplitudes are well approximated by the Rayleigh distribution (Ochi 2005; Holthuijsen 2007). However, because ocean waves are inherently (weakly) nonlinear (Trulsen 2018), wave-wave interactions or other mechanisms can result in considerable deviations from the Gaussian model (Fedele et al. 2016). Several authors have suggested that rogue waves may be a result of non-equilibrium dynamics: if waves are somehow forced into an unstable state, their statistics can deviate in such a way as to suggest an increased likelihood of extreme events (Janssen and Herbers 2009; Viotti and Dias 2014). The kurtosis of the free surface elevation is a convenient metric by which to quantify such deviations: an increase in free surface kurtosis signifies an increase in the probability of rogue wave occurrence (Onorato et al. 2004; Mori and Janssen 2006).

Waves propagating into shallower water are known to be transformed by shoaling and nonlinear effects (Dean and Dalrymple 1991; Dingemans 1997) but recent studies have shown that sudden transitions between deeper and shallower domains can also produce strongly non-Gaussian wave statistics. Physical experiments by Trulsen et al. (2012), Zhang et al. (2019), and Trulsen et al. (2020) showed significant increases in free surface skewness and kurtosis for irregular waves near the crest of an inclined seabed of 1-in-20 slope connecting otherwise flat domains, and these findings have been supported by numerical simulations due to Sergeeva et al. (2011), Gramstad et al. (2013), Viotti and Dias (2014), Ducrozet and Gouin (2017), Zhang et al. (2019), and Zheng et al. (2020). Similar results have also been obtained in experimental and numerical studies of waves propagating over submerged bars (Ma et al. 2014, 2015), shoals (Janssen and Herbers 2009; Raustøl 2014; Fallahi 2016; Trulsen et al. 2020), compound slopes (Kashima et al. 2014), and vertical steps (Zheng et al. 2020).
The foregoing local increases in skewness and kurtosis usually coincide with local enhancements of higher harmonic content related to the sudden decreases in depth and corresponding increases in nonlinearity (Gramstad et al. 2013; Zhang et al. 2019; Trulsen et al. 2020). In fact, Zheng et al. (2020) have recently shown that second-order terms in wave steepness are responsible for the change in the statistical properties near the depth transition for the cases examined by Trulsen et al. (2012) and Gramstad et al. (2013). These deviations are also expected to depend on the initial steepness, spectral bandwidth, and directionality of the waves (Ducrozet and Gouin 2017; Støle-Hentschel et al. 2018; Trulsen et al. 2020; Zheng et al. 2020), the gradient of the seabed slope, and the depth beyond the slope: for milder slopes and deeper depths beyond the slopes, there may be no local maxima, or perhaps even local minima, in skewness and kurtosis (Zeng and Trulsen 2012; Gramstad et al. 2013; Raustøl 2014; Fallahi 2016; Trulsen et al. 2020).

In this paper, the phenomenon of increased free surface skewness and kurtosis following a sudden depth transition is explored further using an accurate yet computationally efficient Boussinesq-type model, following the work of Gramstad et al. (2013), whose model appears to be the simplest of those describing such anomalous statistical deviations. The aim is to first reproduce the findings of Trulsen et al. (2012) and Gramstad et al. (2013) and then extend the parameter space in the numerical simulations to provide further insight into the underlying physics. The paper is structured as follows: Sect. 2 provides a brief description of the numerical model, set-up of the numerical simulations, and grid convergence and sponge layer calibration tests; Sect. 3 compares the present findings with those of Trulsen et al. (2012) and Gramstad et al. (2013) and summarises the results of a model parameter study; and Sect. 4 presents the discussion, conclusions, and recommendations for further work.

\section{Model}

\subsection{Numerical model}

The present simulations are performed using OXBOU, a depth-integrated hybrid numerical model designed to simulate the propagation in one horizontal dimension of ocean surface gravity waves from intermediate to shallow and zero water depth. A brief overview of the model features will suffice here; detailed descriptions of the numerical implementation and verification and validation tests are given by Orszaghova (2011), Orszaghova et al. (2012), and Fitzgerald et al. (2016).

The OXBOU model uses two sets of governing equations and two numerical schemes: unbroken waves are simulated using weakly dispersive, weakly non-linear Boussinesq-type 
equations, which are solved using a fourth-order finite difference method, whilst broken waves are modelled as bores using the non-dispersive, non-linear shallow water equations, which are solved using a shock-capturing finite volume scheme (Orszaghova et al. 2012). The model switches from the Boussinesq-type to shallow water equations when certain depth or free-surface slope criteria are met, but the present simulations involve non-breaking waves solely and so employ only the Boussinesq-type model. The numerical scheme incorporates a moving boundary piston paddle wavemaker, which is facilitated by a mapping between stretchingcompressing physical and fixed computational sub-domains, and is capable of producing waves with approximately correct second-order bound harmonics (see Orszaghova et al. 2012). The scheme also includes an absorbing-generating sponge layer which allows incident waves to propagate freely inshore whilst simultaneously removing offshore-travelling reflections (see Fitzgerald et al. 2016).

OXBOU solves the Boussinesq-type equations of Madsen and Sørensen (1992), which were selected for their enhanced linear dispersion characteristics and computational efficiency (Borthwick et al. 2006; Orszaghova et al. 2012). Following Orszaghova et al. (2012) and Fitzgerald et al. (2016), these equations are presented in a well-balanced, stage-discharge $(\eta, q)$ form as

$$
\begin{aligned}
\eta_{t} & +q_{x}=\psi\left(\eta_{o}-\eta\right), \\
q_{t}+ & \left(\frac{q^{2}}{d}+\frac{1}{2} g\left(\eta^{2}-2 \eta b\right)\right)_{x} \\
= & -g \eta b_{x}-\frac{\tau_{b}}{\rho}+\frac{1}{3} h^{2} q_{x x t}+\frac{1}{3} h h_{x} q_{x t} \\
& +B\left(h^{2} q_{x x t}+g h^{3} \eta_{x x x}+2 g h^{2} h_{x} \eta_{x x}\right) \\
& +\psi\left(q_{o}-q\right),
\end{aligned}
$$

where $\eta=b+h+\zeta$ is the free surface elevation above a prescribed horizontal datum (with $b$ the depth of the datum below the seabed, $h$ the still water depth, and $\zeta$ the free surface elevation above still water level); $q$ is depth-integrated velocity; $\psi$ is the sponge layer damping strength; $d=h+\zeta$ is the total depth; $g$ is acceleration due to gravity; $\tau_{b}$ is bed stress; $\rho$ is the fluid density; the subscripts $t$ and $x$ denote partial derivatives with respect to time and horizontal distance, respectively; the subscript $o$ refers to solutions imposed by the sponge layers; and $B$ is a linear dispersion coefficient such that the wave celerity, $c$, is given by

$$
\frac{c^{2}}{g h}=\frac{1+B k^{2} h^{2}}{1+\left(B+\frac{1}{3}\right) k^{2} h^{2}},
$$

where $k$ is the wave number. Setting $B=1 / 15$ embeds the [2,2] Padé approximant of the exact linear dispersion relation within the momentum equation, whereas setting $B=0$ recovers the classical equation derived by Peregrine (1967).

\subsection{Set-up of numerical simulations}

Following Gramstad et al. (2013), the first set of simulations is designed to replicate the physical experiments described by Trulsen et al. (2012), which were performed in the shallow water basin at the Maritime Research Institute Netherlands (MARIN). These experiments considered three cases of long-crested irregular waves propagating from a piston-type wavemaker (at $x=0 \mathrm{~m}$ ) first over a deeper flat domain, then over a 1-in-20 inclined seabed slope (from $x=143.41$ to $149.41 \mathrm{~m}$ ), and finally over a shallower flat domain leading to an absorbing beach (at $x=173.41 \mathrm{~m}$ ). In all three experimental cases, the still water depths before and after the slope were $h=0.6$ and $0.3 \mathrm{~m}$, respectively, and the nominal input significant wave height was $H_{s}=0.06 \mathrm{~m}$. Cases 1, 2 and 3 were distinguished by the nominal peak periods of their input wave spectra: $T_{p}=1.27,1.70$, and $2.12 \mathrm{~s}$, respectively. Wave records were obtained from eight gauges placed along the length of the basin, and the influence of the depth transition on the probability of rogue wave occurrence was examined by calculating the skewness and kurtosis of the free surface elevation and exceedance function of the (Hilbert) wave envelope at each location.

In repeating these experiments, the present study follows closely the methodology described by Trulsen et al. (2012) but uses OXBOU to output results at $1 \mathrm{~m}$ spatial intervals, and moves the seabed slope $0.01 \mathrm{~m}$ closer to the wavemaker to facilitate the use of uniform (fixed) computational grids. The simulations for each case are performed as follows. The wavemaker is used to generate identical irregular waves in both an incident domain and a run-up domain. In the incident domain, the numerical wave tank (from $x=0 \mathrm{~m}$ to $200 \mathrm{~m}$ ) is assigned a flat seabed profile $(h=0.6 \mathrm{~m})$, whilst in the run-up domain, the tank comprises deeper $(h=0.6 \mathrm{~m})$ and shallower $(h=0.3 \mathrm{~m})$ sections connected by a 1 -in-20 seabed slope (from $x=143.4$ to $149.4 \mathrm{~m}$ ). In both domains, the bed is frictionless and the waves propagate into an absorbing sponge layer (from $x=185.8$ to $200 \mathrm{~m}$ ), which gradually reduces $\zeta$ and $q$ to zero to ensure that there are no reflections either from the end of the tank or the absorbing layer itself. Meanwhile, in the run-up domain, reflections from the slope are removed by an additional absorbing-generating sponge layer (from $x$ $=92.9$ to $107.1 \mathrm{~m}$ ), which adjusts the free surface elevation, $\zeta_{r}$, and depth-integrated velocity, $q_{r}$, to match those in the incident domain, $\zeta_{i}$ and $q_{i}$ (Fig. 1).

Irregular waves are produced as the sum of wave components obtained from a truncated JONSWAP spectrum with peak frequency $f_{p}=1 / T_{p}$ and upper and lower cut-off 


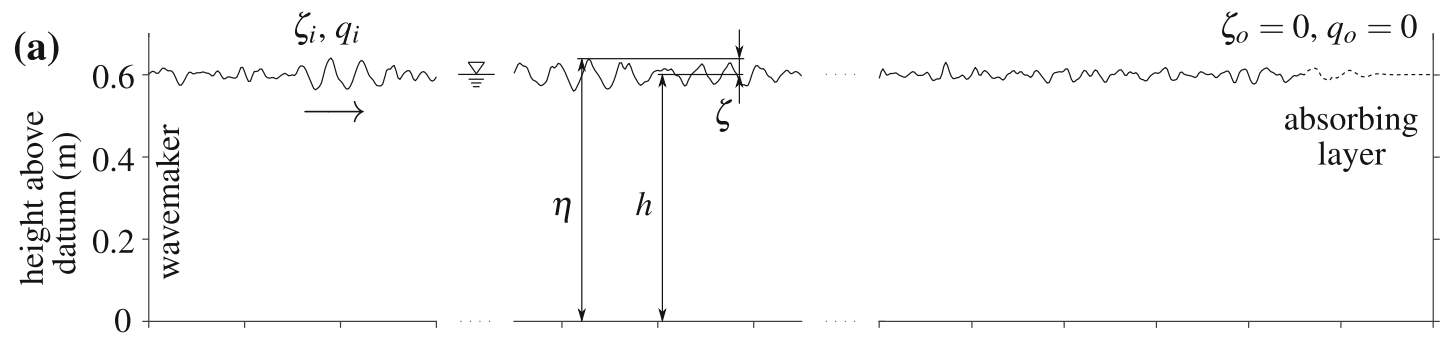

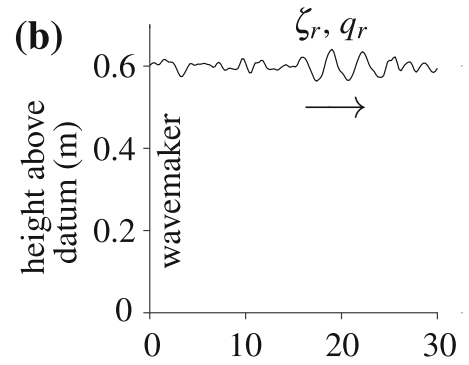

Fig. 1 Schematic diagram showing a simulation performed using coupled (a) incident and (b) run-up domains. Identical irregular waves are produced by the moving boundary wavemakers (left), and absorb-

frequencies $f_{\max }=3 f_{p}$ and $f_{\min }=0.5 f_{p}$. The JONSWAP function is given by

$$
\begin{aligned}
S(f)= & \alpha \frac{g^{2}}{(2 \pi)^{4}} \frac{1}{f^{5}} \exp \left\{-1.25\left(f_{p} / f\right)^{4}\right\} \\
& \gamma^{\exp \left\{-\left(f-f_{p}\right)^{2} / 2\left(\sigma f_{p}\right)^{2}\right\}},
\end{aligned}
$$

where $f$ is the component frequency, $\alpha$ is the energy scale parameter, $\gamma=3.3$ is the peak shape parameter, and $\sigma$ is the peak width factor, which is assigned values of $\sigma=0.07$ for $f \leq f_{p}$ and $\sigma=0.09$ for $f>f_{p}$ (Ochi 2005; Holthuijsen 2007). Pseudo-random wave signals are generated using the random-amplitude/random-phase approach of Tucker et al. (1984), in which the amplitudes and phases of the linear components are determined, respectively, from a Rayleigh distribution with scale parameter $\sqrt{S(f) \triangle f}$, where $\Delta f$ is the frequency domain sampling interval, and a uniform distribution on $[0,2 \pi]$ (Fitzgerald et al. 2016). The corresponding linear wavemaker signal is then calculated using the Biésel transfer function, and a large number of harmonic components is chosen to ensure that the repeat period of the signal is greater than the duration of the simulation. This linear signal can also be corrected by applying a secondorder transfer function approximated from the wavemaker theory of Schäffer (1996) but, for ease of computation, only first-order accurate wavemaker signals are considered initially.

\subsection{Grid convergence and sponge calibration tests}

Model solutions converged for a uniform computational grid spacing of $0.02 \mathrm{~m}$ and a time step of $\sim 0.0066 \mathrm{~s}$. Figure $2 \mathrm{a}$ shows the excellent agreement in free surface time series obtained when computational grids of resolution $0.018 \mathrm{~m}$, $0.02 \mathrm{~m}$, and $0.022 \mathrm{~m}$ (which reproduce the tank using 11,000 , 10,000 , and 9,000 grid points, respectively) are used to simulate an example focused wave group, which is created by bringing 128 harmonic wave components from the Case 2 spectrum to a linear focus amplitude of $0.03 \mathrm{~m}$ at the toe of the seabed slope $(x=143.4 \mathrm{~m})$. Wave records from a point just beyond the crest of the slope $(x=150 \mathrm{~m})$ show excellent agreement, with root mean square error (RMSE) values ranging from $\sim 2.47 \times 10^{-5} \mathrm{~m}$ to $\sim 5.68 \times 10^{-5} \mathrm{~m}$, as do the corresponding frequency-domain results, which are not shown for brevity. Excellent results are also obtained in tests for mass conservation, reversibility, and the accumulation of round-off error, with model errors typically much less than $1 \%$.

The absorbing and absorbing-generating sponge layers are then calibrated to ensure that they are able to damp effectively waves passing through without altering the incoming wave field. The absorbing-generating layer, which is used only in the run-up domain and placed such that its midpoint lies halfway along the one-dimensional tank (Fig. 1), is assigned a triangular strength profile (such that $\psi$ increases and decreases linearly and symmetrically about the midpoint of the layer), whilst the identical absorbing layers, which are 


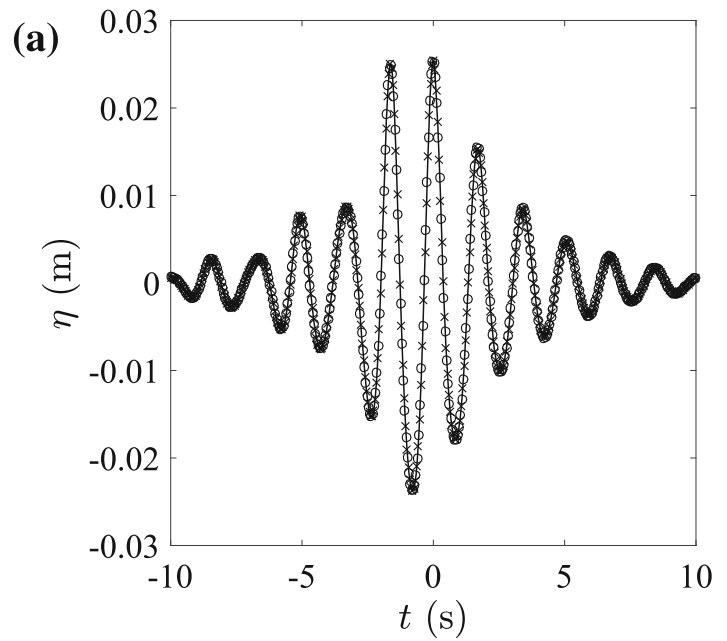

Fig. 2 Free surface elevation time histories at $x=150 \mathrm{~m}$ showing excellent agreement between (a) records of a crest-focused group simulated on computational grids of resolution $0.018 \mathrm{~m}$ (circles), $0.02 \mathrm{~m}$ (line),

placed at the ends of the tanks in both the incident and runup domains, are given linearly increasing strength profiles (Fitzgerald et al. 2016).

Calibration is undertaken by comparing, for different sponge layer lengths, $L_{s}$, and integrated sponge layer strengths, $\bar{\psi}$, the wave records obtained from points upstream and downstream of the sponge layers. With the absorbinggenerating layer switched off, a crest-focused wave group is first propagated from left to right through the absorbing layers, which are temporarily moved $20 \mathrm{~m}$ upstream so that measurements can be taken both upstream and downstream of the layers, and measurements are taken in the run-up domain as the waves are damped to zero. With the absorbing layers calibrated and moved back to the end of the tank, the reflected wave group, which is obtained from an additional simulation with no sponge layers, is then propagated from right to left through the absorbing-generating layer, which is set to damp the waves to the conditions in the incident domain (in this case, still water).

Excellent absorption properties are achieved by setting, for all layers, $L_{s}=4 \lambda_{p}=14.2 \mathrm{~m}$ and $\bar{\psi}=4 \omega_{p}=$ $14.8 \mathrm{rad} / \mathrm{s}$, where $\lambda_{p}$ is the the peak wavelength and $\omega_{p}$ is the peak angular frequency of the Case 2 spectrum. Following Fitzgerald et al. (2016), a periodic irregular wave signal with repeat period $\sim 2.17 \times 10^{2} \mathrm{~s}$ is then used to determine the efficacy of the sponge layer absorption by testing for repeatability in the wave record at a given gauge. Figure $2 b$ shows the excellent agreement $\left(\mathrm{RMSE} \approx 2.64 \times 10^{-4} \mathrm{~m}\right.$ ) in free surface time series obtained between subsequent repeat periods in the wave record at $x=150 \mathrm{~m}$ in the run-up domain,

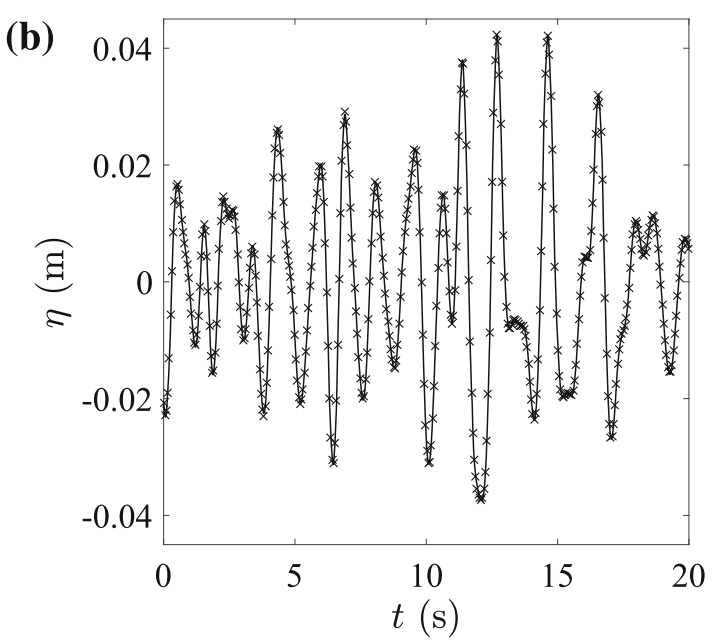

and $0.022 \mathrm{~m}$ (crosses), and (b) subsequent repeat periods (crosses, line) of a periodic irregular wave signal

which confirms that the reflections from the end of the tank and submerged seabed slope are negligible.

\section{Results}

\subsection{Comparison with the results of Trulsen et al. (2012) and Gramstad et al. (2013)}

The three experimental cases performed at MARIN are simulated by first discretising their input spectra into $2^{14}$ harmonic wave components to produce irregular wave signals and corresponding linear paddle signals with repeat periods $\sim 1.67 \times 10^{4} \mathrm{~s}, 1.11 \times 10^{4} \mathrm{~s}$, and $1.39 \times 10^{4} \mathrm{~s}$, respectively (Fig. 3a-b). OXBOU is then used to run each simulation for a duration of $T_{d}=1.10 \times 10^{4} \mathrm{~s}$ with the linear dispersion coefficient tuned for optimal dispersion: $B=1 / 15$. With the three simulations complete, the wave records are compiled and the first $200 \mathrm{~s}$ of each is neglected, following Trulsen et al. (2012), which leaves, at each grid point, records of duration $\sim 8.48 \times 10^{3}, 6.36 \times 10^{3}$, and $5.90 \times 10^{3}$ peak wave periods, respectively. Figure $3 \mathrm{c}$ shows, for the Case 2 simulation, the convergence of the normalised mean, standard deviation, skewness, and kurtosis of the free surface elevation with number of time samples in the wave record at $x=150 \mathrm{~m}$. Each statistic is normalised by the corresponding value obtained for the entire record, and it is clear that the $\sim 1.644 \times 10^{6}$ samples are sufficient to provide robust estimates for each experimental case.

Figure 4 then compares, for each case, the simulated variations in variance, skewness, and kurtosis along the length 

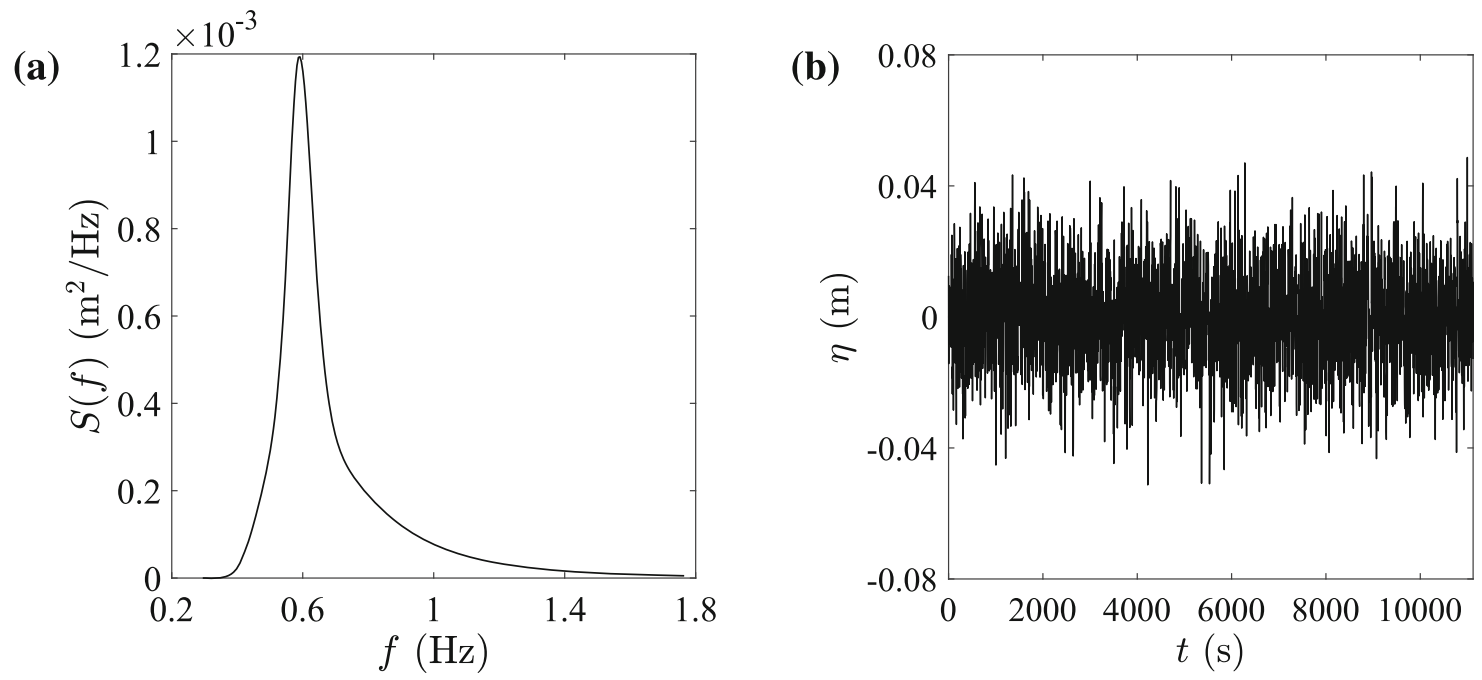

(c)

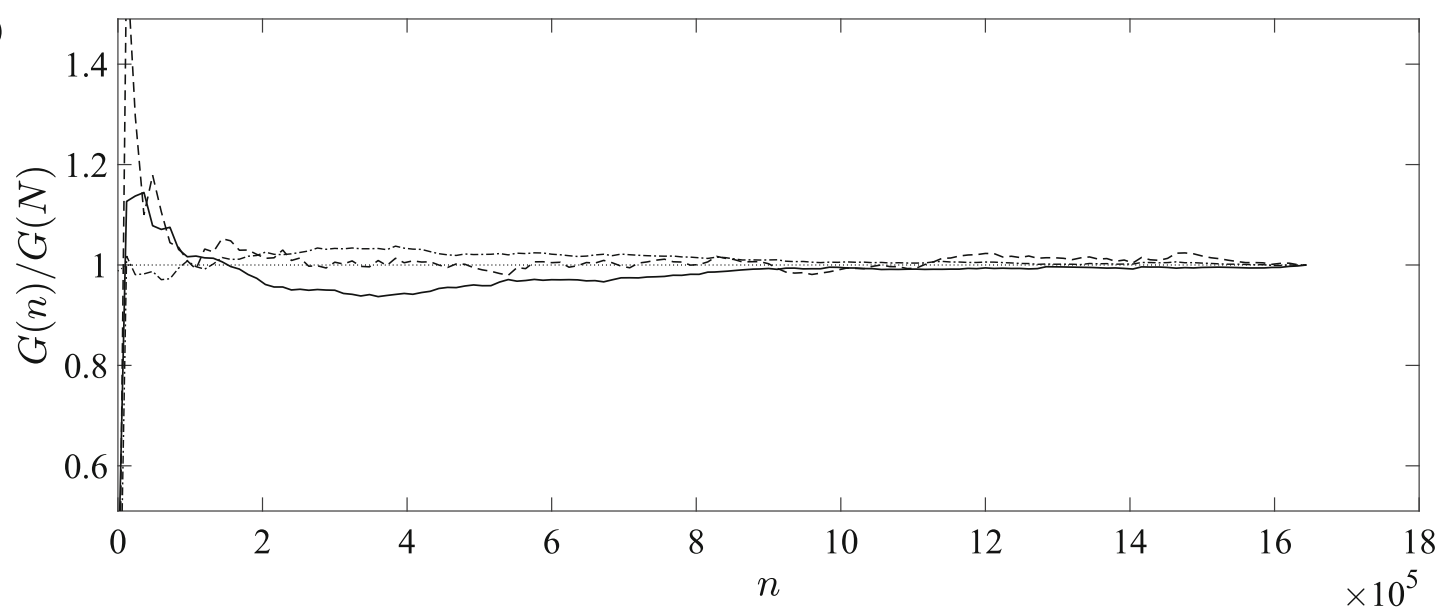

Fig. 3 Example plots from the present Case 2 simulation showing (a) the input JONSWAP spectrum, (b) the nominal input wave signal, and (c) the convergence of the statistical moments $G$ with the number of time samples $n$ in the wave record (which has a total of $N \approx 1.644 \times 10^{6}$ samples) at $x=150 \mathrm{~m}$ : mean (dotted line), standard deviation (dasheddotted line), skewness (dashed line), and kurtosis (solid line) of the tank with those obtained from the Boussinesq-type numerical simulations of Gramstad et al. (2013) and the physical experiments of Trulsen et al. (2012). The results from the present Boussinesq-type simulations are shown with $95 \%$ confidence intervals determined using histograms produced by calculating the same statistics for 1000 bootstrap samples, which are obtained by random sampling with replacement of $5 \%$ of the available data. Although the trends for each statistic are qualitatively similar, the present profiles do not match those reported by Trulsen et al. (2012) and Gramstad et al. (2013): the skewness results are consistently lower and initially negative (Fig. 4d-f), and the kurtosis profiles exhibit greater reductions along the tank and much less prominent spikes near the crest of the submerged seabed slope (Fig. 4g-i).

\subsection{Case 2 parameter study}

To investigate these discrepancies, a parameter study based on the Case 2 simulation is used to examine the effects of various model inputs on the kurtosis profiles obtained for irregular waves propagating over a flat, horizontal bed (Fig. 5a) as well as over the submerged seabed slope (Fig. 5b). For a flat domain with still water depth $h=0.6 \mathrm{~m}$, the kurtosis profile obtained for $x<143.4 \mathrm{~m}$ (Fig. 5a: solid line) is practically identical to that obtained in the Case 2 simulation (Fig. 5b: solid line), which confirms that the upstream kurtosis profile is unaffected by reflections from the submerged slope. This flat-bed simulation also demonstrates a reduction in kurtosis along the length of the tank: the kurtosis decreases from the input value of $\sim 3$ and appears to stabilise at a value of $\sim 2.9$ towards the end of the domain. Repeating this sim- 


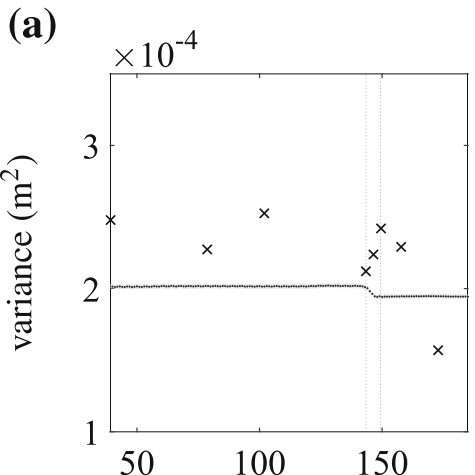

(d)

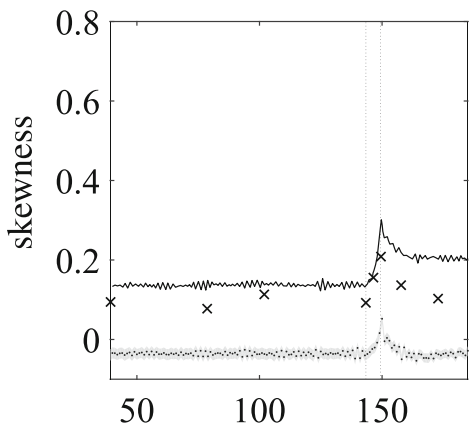

(g)

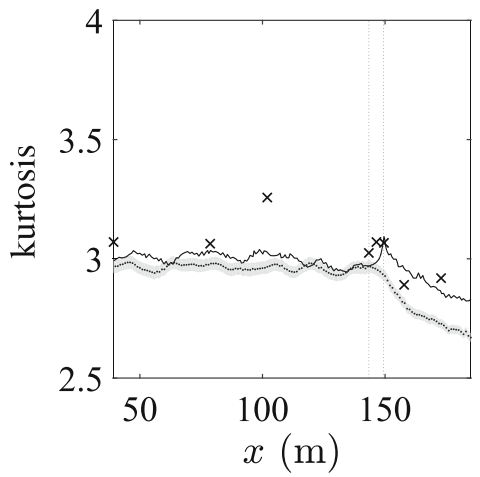

(b)

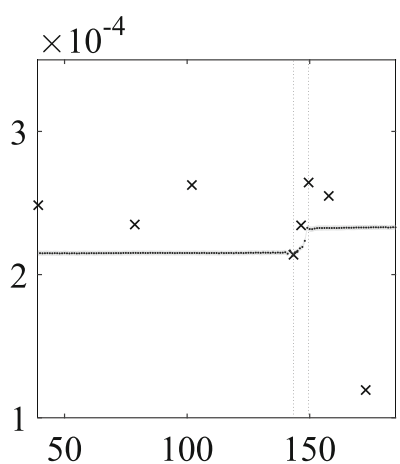

(e)

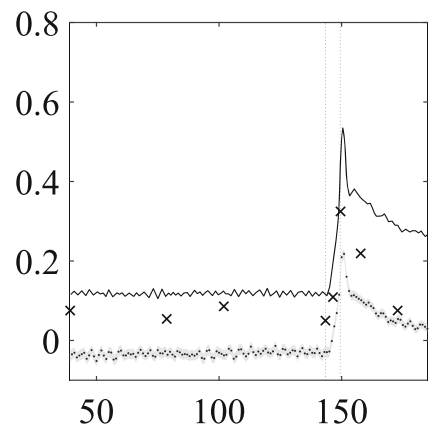

(h)

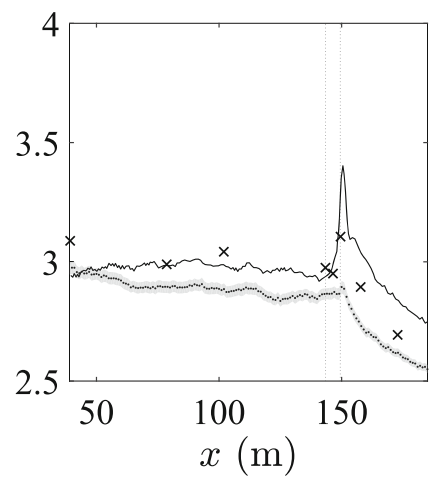

(c)

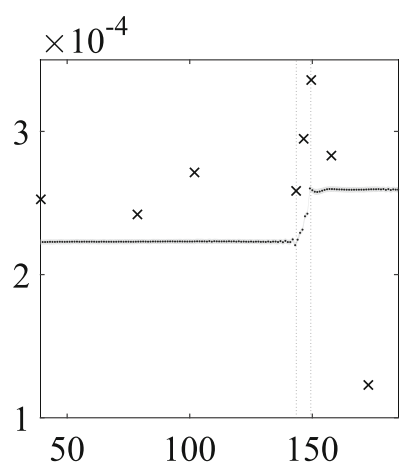

(f)

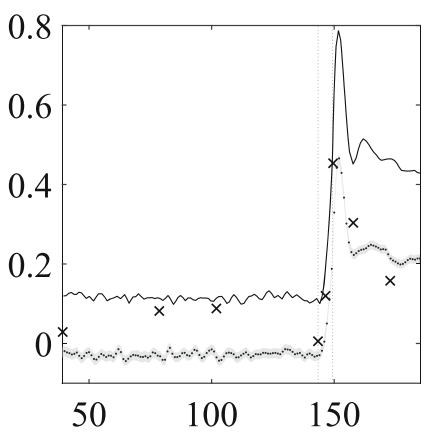

(i)

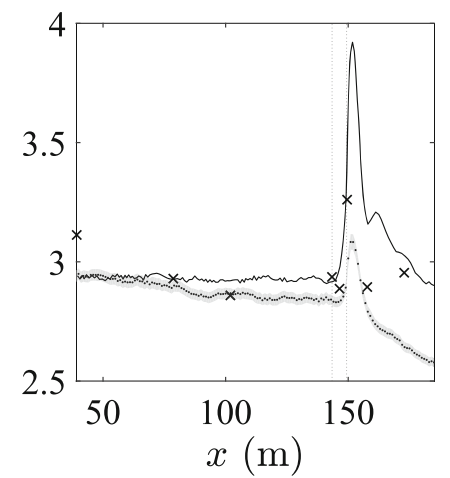

Fig. 4 Profiles of free surface elevation statistics: variance $(\mathbf{a}-\mathbf{c})$, skewness (d-f), and kurtosis (g-i) for Cases 1 (left column), 2 (centre column), and 3 (right column). Results are obtained from the physical experiments of Trulsen et al. (2012) (crosses), the Boussinesq- type simulations of Gramstad et al. (2013) (solid lines), and the present Boussinesq-type simulations (dots with 95\% confidence intervals shaded in grey). The vertical dotted lines mark the positions of the toe (left) and crest (right) of the submerged seabed slope ulation with a lower input value of kurtosis (which is done by replacing the original wavemaker signal with the negatively skewed wave record subsequently obtained at $x=160 \mathrm{~m}$ ) yields a more uniform profile, which further suggests an equilibrium kurtosis value of $\sim 2.9$ for this case. However, this equilibrium value is found to depend, as in earlier studies (see Janssen 2003; Zeng and Trulsen 2012), on both the still water depth (Fig. 5a: dotted line) and the bandwidth of the input wave spectrum (Fig. 5a: dashed line).

For simulations including the submerged seabed slope, the kurtosis profiles appear insensitive to the location of the generating-absorbing sponge layer and the end-of-tank boundary condition. A similar profile is also obtained when the strengths of the absorbing and absorbing-generating layers are reduced by $90 \%$ (Fig. 5b: dotted line), which implies that the observed reduction in kurtosis is not the result of excess numerical damping. Dividing each wave record from the Case 2 simulation into five equal sections and taking the quasi-ensemble average of these fifths yields a similar profile (Fig. 5b: dashed line), as does taking the ensemble average across five alternate, independent realisations (Fig. 5b: dashed-dotted line). This demonstrates that the 


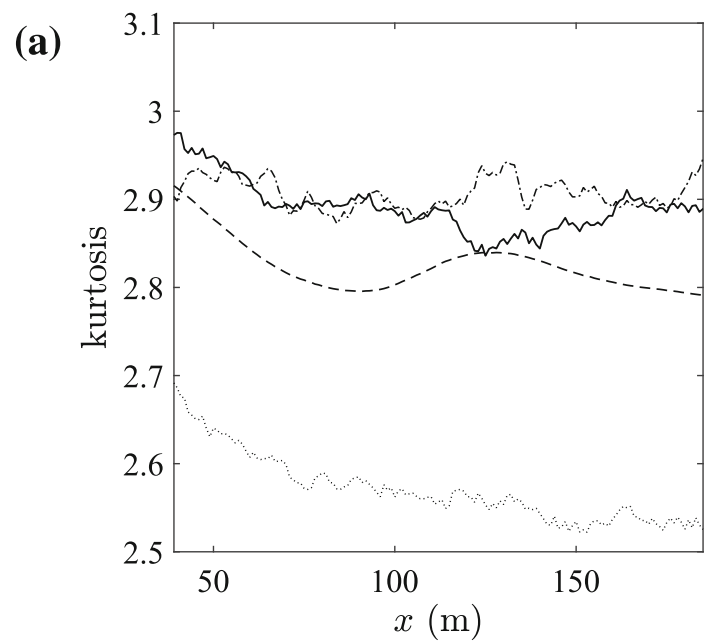

Fig. 5 Kurtosis profiles from the Case 2 parameter study. (a) Flat domain: still water depth, $h=0.6 \mathrm{~m}$ (solid line); narrower input spectrum (dashed line); lower input kurtosis (dashed-dotted line); and $h=0.3 \mathrm{~m}$ (dotted line). (b) Submerged seabed slope: single realisation (solid line); quasi-ensemble average of the single realisation divided

present results do not depend on the type of measurement taken. Moreover, the kurtosis profiles obtained from shorterduration (for ease of computation) simulations using firstand second-order accurate wavemaker signals are very similar (Fig. 5b: circles; crosses), which implies that neither are the observed trends due to error waves produced by the firstorder accurate wavemaker (see Orszaghova et al. 2014).

\section{Discussion and conclusions}

The kurtosis profiles obtained in each experimental case agree qualitatively with those of Trulsen et al. (2012) and Gramstad et al. (2013) but the present numerical model is clearly unable to capture accurately the spikes near the crests of the submerged seabed slopes (Fig. 4g-i). A parameter study has confirmed that the present results do not depend on the type of measurement taken, the position or damping strengths of the sponge layers, or the order of accuracy of the wavemaker signal (Fig. 5b). Further discrepancies are also evident: for the depths considered here, second-order bound harmonics are expected to positively skew the probability distribution function for the free surface elevation (Onorato et al. 2005) but the present skewness results are initially negative (Fig. 4d-f). Replication of an example irregular wave simulation with the 'fully nonlinear' OceanWave3D model (see Engsig-Karup et al. 2009) (comparison not shown for brevity) confirms that OXBOU produces consistently lower values of free surface elevation skewness and kurtosis. (b)

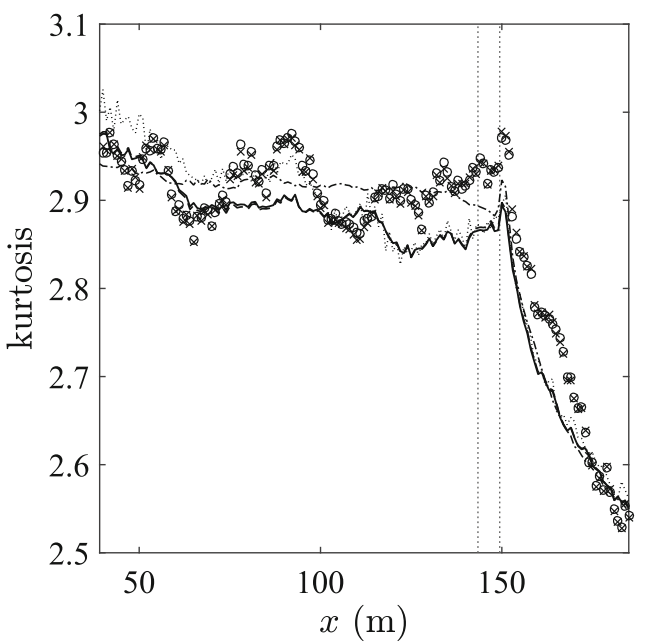

into fifths (dashed line); ensemble average of five alternate, independent realisations (dashed-dotted line); reduced sponge layer strengths (dotted line); and shorter simulations using first- (circles) and secondorder (crosses) accurate wavemaker signals

The discrepancies between the present results and those of Gramstad et al. (2013) most likely stem from differences in the underlying momentum equations. The exact source of these discrepancies, however, is difficult to determine. When examining the propagation of irregular waves over a compound slope, Kashima et al. (2014) found that the present equation set returned values of skewness and kurtosis which were considerably lower than those obtained in the corresponding physical experiment. These lower values were explained as being the result of insufficient nonlinearity in the numerical simulations, but Gramstad et al. (2013) were able to use a similar weakly nonlinear model to reproduce the results of Trulsen et al. (2012). Further, in deriving the present equation set, Madsen and Sørensen (1992) adopted a mild slope assumption which retained only the lowest-order spatial derivatives of the water depth. This means that the present model is unable to capture the effects of the sudden depth transition as well as that of Gramstad et al. (2013), which retains these high-order terms. It is also worth noting that two of the present three experimental cases consider water depths which exceed the depth limit $\left(k_{p} h<1\right.$, where $k_{p}$ is the peak wavenumber of the input spectrum) recommended to ensure the accuracy of the present equation set (see Madsen and Sørensen 1992, 1993).

Using a boundary element method with fast multipole acceleration to solve Laplace's equation for potential flow with fully nonlinear boundary conditions, Zheng et al. (2020) have recently predicted the local changes in the statistical properties of irregular waves propagating over a range of submerged slopes in close agreement with the experiments 
by Trulsen et al. (2012). In doing so, Zheng et al. (2020) have demonstrated that these local changes are driven by second-order terms, which may help to explain why the peaks in skewness and kurtosis cannot be accurately captured by the present Boussinesq-type model. The present equation set includes a linear dispersion coefficient, $B$, which may be tuned to produce either enhanced dispersion characteristics or approximately correct second-order bound harmonics (Yao 2007). Herein, $B$ is assigned a value of $1 / 15$ for optimal dispersion. It is reasonable to assume that if the bound waves are inaccurate, significant errors in skewness and kurtosis will arise near the sudden depth transition, because the peaks in skewness and kurtosis at this location are likely a consequence of the release of second-order bound waves by the depth transition (Zheng et al. 2020). Although there is no value of $B$ which can make the present equation set equivalent to that of Gramstad et al. (2013), it is possible to match the linear dispersion relations by setting $B=0.057$. However, this is found to make no appreciable difference to the present results and does not address the need to correct the bound waves. Frequency domain comparisons between OceanWave3D and OXBOU (again not shown for brevity) demonstrate that there is also no value of $B$ which gives satisfactory agreement on sub-harmonic and super-harmonic content.

Modelling this sudden depth transition problem is challenging because it requires an accurate yet computationally efficient numerical code which is able to incorporate the effects of both dispersion and nonlinearity on the evolution of the wave field. The work of Gramstad et al. (2013) has shown that the key physics underlying this localised increase in the probability of rogue wave occurrence can be captured by a weakly dispersive, weakly nonlinear Boussinesq-type model. There are, however, many different sets of Boussinesq-type equations and the present study demonstrates the importance of making an appropriate selection. Although OXBOU is a very useful tool for modelling nearshore wave propagation, run-up, and overtopping, it is clear that the underlying equation set is not well-suited to modelling the propagation of waves over a sudden depth transition. It is thus recommended that this problem be revisited using a revised version of OXBOU based on an improved set of Boussinesq-type equations. The equations of Schäffer and Madsen (1995), for instance, provide the same enhanced linear dispersion characteristics as those of Madsen and Sørensen (1992) but are not limited to mildly sloping seabeds. It should also be noted, however, that the accuracy of any numerical model will depend on the means by which the spatial and temporal derivatives are calculated (Borthwick et al. 2006), and that sudden depth transitions invariably prove challenging for any low-order finite difference scheme. Shock-capturing schemes offer an alternative approach but are generally less accurate and may introduce further complications.
In future studies, it would prove valuable to compare statistical results not only between different Boussinesq-type formulations but also between weakly and highly nonlinear models, following Viotti and Dias (2014), Ducrozet and Gouin (2017), and Zheng et al. (2020), as well as with physical experiments, following Zhang et al. (2019) and Trulsen et al. (2020). It would also be interesting to explore whether idealised, multi-layer numerical models, such as SWASH (Zijlema et al. 2011), can provide additional insight. Future work should examine not only the extreme amplitudes but also the shapes and periods of these rogue waves, which are crucial in understanding the strength of the wave impact and the resilience of ships and offshore structures (Kharif et al. 2009; Adcock and Taylor 2014). The effects of directionality must also be considered because large waves evolve differently in unidirectional and directionally spread seas (Adcock and Taylor 2014), and studies have shown that even a small amount of counter-propagating wave energy can result in a significant reduction in free surface kurtosis (Ducrozet and Gouin 2017; Støle-Hentschel et al. 2018). Finally, real-world observations should be included wherever possible in studies of rogue wave formation and occurrence probability (Slunyaev et al. 2011) because it is the ocean that provides the most representative conditions with which to test and revise new theories.

Acknowledgements The authors gratefully acknowledge support from the UK's Engineering and Physical Sciences Research Council (EPSRC) and Natural Environment Research Council (NERC), which sponsored this research under grant number EP/R007632/1. The authors wish to thank Dr Jana Orszaghova and Prof. Paul H. Taylor, who contributed greatly to the development of the OXBOU model; Tianning Tang, who carried out OceanWave3D simulations to compare with the present results; Prof. Vengatesan Venugopal, for his support during the later stages of the project; and three anonymous reviewers for their helpful comments. PAJB also wishes to thank Drs Tim Bunnik, Jacob Dobson, Samuel Draycott, Frances M. Judge, Yan Li, James N. Steer, and James Young for providing much valuable information and many helpful discussions. TSvdB was supported by a Royal Academy of Engineering Research Fellowship.

Open Access This article is licensed under a Creative Commons Attribution 4.0 International License, which permits use, sharing, adaptation, distribution and reproduction in any medium or format, as long as you give appropriate credit to the original author(s) and the source, provide a link to the Creative Commons licence, and indicate if changes were made. The images or other third party material in this article are included in the article's Creative Commons licence, unless indicated otherwise in a credit line to the material. If material is not included in the article's Creative Commons licence and your intended use is not permitted by statutory regulation or exceeds the permitted use, you will need to obtain permission directly from the copyright holder. To view a copy of this licence, visit http://creativecomm ons.org/licenses/by/4.0/. 


\section{References}

Adcock TAA, Taylor PH (2014) The physics of anomalous ('rogue') ocean waves. Rep Prog Phys 77:105901. https://doi.org/10.1088/ 0034-4885/77/10/105901

Adcock TAA, Taylor PH, Yan S, Ma QW, Janssen PAEM (2011) Did the Draupner wave occur in a crossing sea? Proc R Soc A 467:30043021. https://doi.org/10.1098/rspa.2011.0049

Borthwick AGL, Ford M, Weston BP, Taylor PH, Stansby PK (2006) Solitary wave transformation, breaking, and run-up at a beach. Proc. ICE - Marit. Eng 159(3):97-105. https://doi.org/10.1680/ maen.2006.159.3.97

Cavaleri L, Barbariol F, Benetazzo A, Bertotti L, Bidlot J-R, Janssen P, Wedi N (2016) The Draupner wave: a fresh look and the emerging view. J. Geophys. Res. Oceans 121:6061-6075. https://doi.org/10. 1002/2016JC011649

Christou M, Ewans K (2014) Field measurements of rogue water waves. J Phys Oceanogr 44(9):2317-2335. https://doi.org/10.1175/JPOD-13-0199.1

Dean RG, Dalrymple RA (1991) Water wave mechanics for engineers and scientists. World Scientific, Singapore

Dingemans MW (1997) Water wave propagation over uneven bottoms. World Scientific, Singapore

Ducrozet G, Gouin M (2017) Influence of varying bathymetry in rogue wave occurrence within unidirectional and directional sea-states. J Ocean Eng Mar Energy 3:309-324. https://doi.org/10.1007/ s40722-017-0086-6

Dysthe K, Krogstad HE, Müller P (2008) Oceanic rogue waves. Ann Rev Fluid Mech 40:287-310. https://doi.org/10.1146/annurev.fluid.40. 111406.102203

Engsig-Karup AP, Bingham HB, Lindberg O (2009) An efficient flexible-order model for 3D nonlinear water waves. J Comp Phys 228(6):2100-2118. https://doi.org/10.1016/j.jcp.2008.11.028

Fallahi S (2016) Freak waves over nonuniform depth with different slopes. Master's thesis, University of Oslo, Norway

Fedele F, Brennan J, Ponce De León S, Dudley J, Dias, F (2016) Real world ocean rogue waves explained without the modulational instability. Sci. Rep. 6: 27715, https://doi.org/10.1038/srep27715

Fitzgerald CJ, Taylor PH, Orszaghova J, Borthwick AGL, Whittaker C, Raby AC (2016) Irregular wave runup statistics on plane beaches: Application of a Boussinesq-type model incorporating a generating-absorbing sponge layer and second-order wave generation. Coast. Engng 114: 309-324, http://dx.doi.org/10.1016/j. coastaleng.2016.04.019

Gramstad O, Zeng H, Trulsen K, Pedersen GK (2013) Freak waves in weakly nonlinear unidirectional wave trains over a sloping bottom in shallow water. Phys Fluids 25(12): 122103, https://doi.org/10. $1063 / 1.4847035$

Haver S, Andersen OJ (2000) Freak waves: Rare realizations of a typical population or typical realizations of a rare population? In: Proceedings of the 10th international offshore and polar engineering conference, Seattle, WA, USA

Holthuijsen LH (2007) Waves in oceanic and coastal waters. Cambridge University Press, Cambridge

Janssen PAEM (2003) Nonlinear four-wave interactions and freak waves. J Phys Oceanogr 33(4): 863-884, https://doi.org/10.1175/ 1520-0485(2003)33<863:NFIAFW>2.0.CO;2

Janssen TT, Herbers THC (2009) Nonlinear wave statistics in a focal zone. J Phys Oceanogr 39(8):1948-1964. https://doi.org/10.1175/ 2009JPO4124.1

Kashima H, Hirayama K, Mori N (2014) Estimation of freak wave occurrence from deep to shallow water regions. Coast Eng Proc 1(34):36. https://doi.org/10.9753/icce.v34.waves.36

Kharif, C, Pelinovsky, E, Slunyaev, A (2009) Rogue waves in the ocean. Springer Science \& Business Media
Ma Y, Dong G, Ma X (2014) Experimental study of statistics of random waves propagating over a bar. Coast. Engng Proc. 1(34):30. https:// doi.org/10.9753/icce.v34.waves.30

Ma Y, Ma X, Dong G (2015) Variations of statistics for random waves propagating over a bar. J Mar Sci Tech 23(6):864-869. https://doi. org/10.6119/JMST-015-0610-3

Madsen, PA, Sørensen, OR (1992) A new form of the Boussinesq equations with improved linear dispersion characteristics, Part 2. A slowly-varying bathymetry. Coast Eng 18(3-4): 183-204, https:// doi.org/10.1016/0378-3839(92)90019-Q

Madsen PA, Sørensen OR (1993) Bound waves and triad interactions in shallow water. Ocean Eng 20(4):359-388. https://doi.org/10. 1016/0029-8018(93)90002-Y

Mori N, Janssen PAEM (2006) On kurtosis and occurrence probability of freak waves. J Phys Oceanogr 36(7):1471-1483. https://doi.org/ $10.1175 / J P O 2922.1$

Ochi MK (2005) Ocean waves: The stochastic approach. Cambridge University Press, Cambridge

Onorato M, Osborne AR, Serio M (2005) On deviations from Gaussian statistics for surface gravity waves. arXiv preprint nlin/0503071, https://arxiv.org/pdf/nlin/0503071.pdf

Onorato M, Osborne AR, Serio M, Cavaleri L, Brandani C, Stansberg CT (2004) Observation of strongly non-Gaussian statistics for random sea surface gravity waves in wave flume experiments. Phys Rev E 70:067302. https://doi.org/10.1103/PhysRevE.70.067302

Onorato M, Residori S, Bortolozzo U, Montina A, Arecchi FT (2013) Rogue waves and their generating mechanisms in different physical contexts. Phys Rep 528(2):47-89. https://doi.org/10.1016/j. physrep.2013.03.001

Orszaghova J (2011) Solitary waves and wave groups at the shore. DPhil thesis, University of Oxford, UK

Orszaghova J, Borthwick AGL, Taylor PH (2012) From the paddle to the beach-a Boussinesq shallow water numerical wave tank based on Madsen and Sørensen's equations. J Comp Phys 231(2):328-344. https://doi.org/10.1016/j.jcp.2011.08.028

Orszaghova J, Taylor PH, Borthwick AGL, Raby AC (2014) Importance of second-order wave generation for focused wave group run-up and overtopping. Coast Eng 94(2):63-79. https://doi.org/10.1016/ j.coastaleng.2014.08.007

Peregrine DH (1967) Long waves on a beach. J Fluid Mech 27(4):815_ 827. https://doi.org/10.1017/S0022112067002605

Raust $\varnothing 1$ A (2014) Freake bølger over variabelt dyp. Master's thesis (in Norwegian), University of Oslo, Norway

Schäffer HA (1996) Second-order wavemaker theory for irregular waves. Ocean Eng 23(1):47-88. https://doi.org/10.1016/00298018(95)00013-B

Schäffer, HA, Madsen, PA (1995) Further enhancements of Boussinesqtype equations. Coast Eng 26(1-2): 1-14, https://doi.org/10.1016/ 0378-3839(95)00017-2

Sergeeva A, Pelinovsky E, Talipova T (2011) Nonlinear random wave field in shallow water: variable Korteweg-de Vries framework. Nat Hazards Earth Syst Sci 11:323-330. https://doi.org/10.5194/ nhess-11-323-2011

Slunyaev A, Didenkulova I, Pelinovsky E (2011) Rogue waters. Contemp Phys 52(6):571-590. https://doi.org/10.1080/00107514. 2011.613256

Støle-Hentschel S, Trulsen K, Rye LB, Raust $\varnothing 1$ A (2018) Extreme wave statistics of counter-propagating irregular long-crested sea states. Phys Fluids 30:067102. https://doi.org/10.1063/1.5034212

Trulsen K (2018) Rogue waves in the ocean, the role of modulational instability, and abrupt changes of environmental conditions that can provoke non equilibrium wave dynamics. In: The ocean in motion. Springer Oceanography

Trulsen K, Raust $\varnothing 1$ A, Jorde S, Rye LB (2020) Extreme wave statistics of long-crested irregular waves over a shoal. J Fluid Mech 882:R2. https://doi.org/10.1017/jfm.2019.861 
Trulsen K, Zeng H, Gramstad O (2012) Laboratory evidence of freak waves provoked by non-uniform bathymetry. Phys Fluids 24(9):097101. https://doi.org/10.1063/1.4748346

Tucker MJ, Challenor PG, Carter DJT (1984) Numerical simulation of a random sea: a common error and its effect upon wave group statistics. Appl Ocean Res 6(2):118-122. https://doi.org/10.1016/ 0141-1187(84)90050-6

Viotti C, Dias F (2014) Extreme waves induced by strong depth transitions: fully nonlinear results. Phys Fluids 26(5):051705. https:// doi.org/10.1063/1.4880659

Yao Y (2007) Boussinesq-type modelling of gently shoaling extreme ocean waves. DPhil thesis, University of Oxford, UK

Zeng H, Trulsen K (2012) Evolution of skewness and kurtosis of weakly nonlinear unidirectional waves over a sloping bottom. Nat Hazards Earth Syst Sci 12(3):631-638. https://doi.org/10.5194/nhess-12631-2012
Zhang J, Benoit M, Kimmoun O, Chabchoub A, Hsu H-C (2019) Statistics of extreme waves in coastal waters: Large scale experiments and advanced numerical simulations. Fluids 4(2):99. https://doi. org/10.3390/fluids4020099

Zheng Y, Lin Z, Li Y, Adcock TAA, Li Y, van den Bremer TS (2020) Fully nonlinear simulations of extreme waves provoked by strong depth transitions: The effect of slope. Phys Rev Fluids 5:064804. https://doi.org/10.1103/PhysRevFluids.5.064804

Zijlema M, Stelling G, Smit P (2011) SWASH: an operational public domain code for simulating wave fields and rapidly varied flows in coastal waters. Coast Eng 58(10):992-1012. https://doi.org/10. 1016/j.coastaleng.2011.05.015

Publisher's Note Springer Nature remains neutral with regard to jurisdictional claims in published maps and institutional affiliations. 